
3 Research Square
Preprints are preliminary reports that have not undergone peer review.
They should not be considered conclusive, used to inform clinical practice,
or referenced by the media as validated information.

\title{
Two Novel PDE11A Genetic Variants Increase Tau Phosphorylations in Early- onset Alzheimer's Disease
}

wei qin

Xuanwu Hospital

Aihong Zhou

Xuanwu Hospital

Xiumei Zuo

Xuanwu Hospital

Longfei Jia

Xuanwu Hospital

Cuibai Wei

Xuanwu Hospital

Yi Tang

Xuanwu Hospital

Fen Wang

Xuanwu Hospital

Fangyu Li

Xuanwu Hospital

Qi Wang

Xuanwu Hospital

Ying Li

Xuanwu Hospital

Yiping Wei

Xuanwu Hospital

Lina Zhao

Xuanwu Hospital

Hongmei Jin

Xuanwu Hospital

Carlos cruchaga

Washington University in Saint Louis

Bruno A. Benitez

Washington University in Saint Louis

jianping Jia ( $\nabla_{\text {jjp@ccmu.edu.cn ) }}$

Xuanwu Hospital, Capital Medical University https://orcid.org/0000-0003-4624-0336

Research article

Keywords: early-onset Alzheimer's disease, phosphodiesterase 11A, Tau phosphorylation, cAMP/PKA pathway

Posted Date: August 20th, 2020

DOI: https://doi.org/10.21203/rs.3.rs-60929/v1

License: (1) (7) This work is licensed under a Creative Commons Attribution 4.0 International License. Read Full License 


\section{Abstract}

Background: Alzheimer's disease (AD) is a leading cause of dementia in the elderly and has become a major health issue. However, a large number of genetic risk factors remain undiscovered.

Methods: To identify novel risk genes and better understand the molecular pathway underlying AD, whole-exome sequencing (WES) was performed in 215 early-onset $A D$ (EOAD) patients and 55 unrelated healthy controls of Han Chinese ethnicity. Subsequent direct sequencing was performed in 4962 individuals to validate the selected rare mutations. Computational annotation and in vitro functional studies were performed to evaluate the role of candidate mutations in $E O A D$ and the underlying mechanisms.

Results: We identified two rare missense mutations in the phosphodiesterase 11A (PDE11A) gene, resulting in p.Arg202His, and p.Leu756GIn, in individuals with EOAD. Both mutations are located in evolutionarily highly conserved amino acids, are predicted to alter the protein conformation, and classified as pathogenic. Furthermore, we found significantly decreased protein levels of PDE11A in brain samples of AD patients. Expression of PDE11A variants and knockdown experiments with specific short hairpin RNA (shRNA) for PDE11A both resulted in an increase of AD-associated Tau hyperphosphorylation at T181, S404, S202, S416, S214, S396 and AT8 epitopes in vitro. PDE11A variants or PDE11A shRNA also caused increased cAMP levels, protein kinase A (PKA) activation, and cAMP response element-binding protein (CREB) phosphorylation. Additionally, pretreatment with a PKA inhibitor (H89) suppressed PDE11A mutation-induced $\mathrm{p}$-Tau formation.

Conclusions: Our results demonstrate that both PDE11A mutations and PDE11A knockdown increase Tau phosphorylation through the cAMP/PKA pathway, suggesting that $P D E 11 A$ is a novel risk gene for AD. This study provides insight into the involvement of Tau phosphorylation via the cAMP/PKA pathway in EOAD pathogenesis and provides a potential new target for intervention.

\section{Background}

Alzheimer's disease (AD) is a leading cause of dementia, affecting between 23 and 35 million people worldwide [1]. AD is one of the major contributors to disability and causes increases in the burdens of patients and the families of patients, as well as of the health care systems [2]. The neuropathologic hallmarks of $A D$ brains are extracellular accumulation of diffuse and neuritic amyloid plaques, composed of amyloid- $\beta$ ( $A \beta$ ) peptide, and the intraneuronal accumulation of neurofibrillary tangles (NFTs) composed of hyperphosphorylated protein tau (p-Tau) [3]. Based on age at onset (AAO), AD is classified as either early onset ( $A A O<65$ years) or late onset ( $A A O \geq 65$ years). Of all AD patients, around $10 \%$ are diagnosed with EOAD. Late-onset $A D$ (LOAD) is a complex disorder with a heterogeneous etiology and a heritability of 58-79\% [4, 5]. Genome-wide association studies (GWAS) have identified over 50 risk loci associated with AD [4]. However, most GWAS loci are noncoding common variants of uncertain function. Indeed, GWAS hits explain a relatively small proportion of the phenotype in populations, and it is estimated that the 'missing heritability' may be explained by rare but functionally important variants. EOAD is an almost entirely genetically determined disease with a heritability ranging $92 \%$ to $100 \%$ [4]. High-penetrant mutations in amyloid precursor protein (APP) and the presenilins 1 and 2 (PSEN1 and PSEN2) are the main genetic risk factors underlying EOAD but are only found in approximately $11 \%$ of all EOAD patients [6]. Whole-exome sequencing (WES) has recently identified rare missense variants in TREM2, ABCA7, CASP7, ADAM10 and other genes that increase the risk for developing AD [7-11]. However, a large number of EOAD patients remain genetically unexplained. EOAD patients are more likely to carry pathogenic variants [4], and suitable for identifying novel $A D$ risk genes. The number of patients with dementia in China accounts for approximately $25 \%$ of the entire population with dementia worldwide [1]. However, very few studies on risk genes in EOAD cohorts have been carried out in China.

In this study, WES was performed in 215 EOAD patients and 55 unrelated healthy controls of Han Chinese ethnicity to identify novel AD risk genes. We identified two rare nonsynonymous variants (p.Arg202His and p.Leu756Gln) in PDE11A in individuals with EOAD. Functional analyses in vitro revealed that both variants enhanced Tau phosphorylation. We also investigated the mechanisms through which PDE11A may be relevant in AD.

\section{Methods}

\section{Study design and subjects}

Data from 215 individuals with EOAD from Xuanwu Hospital were evaluated in this observational study and compared with healthy cognitively normal controls (Additional file 1: Table S1). Each individual underwent a neuropsychological examination, magnetic resonance imaging (MRI); cerebrospinal fluid analysis was performed for a subset of individuals $(n=115)$ in which levels of $A \beta 42, A \beta 40$, Tau and $p$-Tau181 were assessed. The criteria for the recruited EOAD patients were set as follows: 『met the National Institute of Neurological and Communicative Disorders and the Stroke and the Alzheimer Disease and Related Disorders Association (NINCDS/ADRA)[12] or the National Institute on Aging-Alzheimer's Association (NIA-AA) diagnostic criteria [13]; 『the onset age of affected individuals was below 55 years; \no known pathogenic mutations in PSEN1, PSEN2, APP, MAPT or GRN genes. Healthy controls were cognitively normal; amnesia was not present, Mini-Mental State Examination (MMSE) scores were higher than the appropriate cutoff for dementia, Clinical Memory Scale scores $\geqq 90$, and global Clinical Dementia Rating scores were equal to 0 . Additional 513 DNA samples from patients with EOAD and 4449 healthy controls were collected at the Xuanwu Hospital from 2015 to 2020 and used for Sanger sequencing. Signed informed consent was provided by all the patients and control subjects. The study protocol was approved and monitored by the Ethics Committee of Xuanwu Hospital.

Relative PDE11A protein levels were quantified using immunoblot in samples from fresh frozen postmortem parietal lobe tissue of six AD patients and six cognitively healthy controls, recruited at Washington University in St. Louis Charles F. and Joanne Knight Alzheimer's Disease Research Center Brain Bank (Knight ADRC) and approved by the institutional review board of Washington University. Written informed consent for brain autopsy was obtained from all participants or their legal representatives. 
Whole-genomic DNA was obtained from the peripheral blood of all participants. Briefly, the exome was enriched using Agilent SureSelect Human All Exon V5 Kit (Agilent Technologies, Santa Clara, CA, USA) and analyzed using an Illumina HiSeq 2500 (150-bp paired-end, Illumina, San Diego, CA, USA). After conducting quality control, high-quality paired-end reads were mapped to the human genome build GRCh37 using Burrows-Wheeler Aligner software. Verita Trekker was employed to identify variants. Enliven $\AA$ and ANNOVAR were utilized to perform annotation for Variant Call Format, and variants were selected according to an autosomal dominant model. Nonsynonymous exonic or close-to splice-site variants with minor allele frequency $<0.01 \%$ were prioritized for analysis. Variant pathogenicity was evaluated using SIFT, Polyphen-2, MutationTaster, M-CAP, CADD, LRT, PROVEAN, DANN, VEST3, fathmm-MKL, GERP, SiPhy, phastCons, and phyloP tools. Candidate variants were prioritized based on OMIM, MGI, GO, KEGG, ACGM, UKBiobank PheWeb web-based tool and the published literature.

\section{Annotation of PDE11A variants}

InterPro was used to predict domain maps of the PDE11A protein [14]. DNAMAN software (Lynnon Corporation, Quebec, Canada) was applied for multiple sequence alignment. A homology model of the wildtype PDE11A structure was built in MODELLER software using the crystal structure of the phosphodiesterase template (PDB: 3IBJ) [15]. Based on the homology modeling structure of wildtype PDE11A, Arg202His- and Leu756Gln-mutated proteins were generated with Pymol software [16]. AMBER software was used for molecular dynamics simulations for wildtype PDE11A and the Arg202His- and Leu756GIn variants [17]. To investigate in which major cell type the PDE11A gene is expressed, we queried the gene in a publicly available single-cell/nuclei RNA-seq dataset of AD cohorts of human brain tissues (http://adsn.ddnetbio.com/) [18].

\section{Generation of PDE11A variants}

Human wild-type PDE11A cDNA (NM_016953) was cloned into the pCDNA3.1-EGFP vector. Site-directed PDE11A mutagenesis (p.Arg202His and p.Leu756GIn) was performed using a KOD-Plus-Mutagenesis kit (Stratagene, La Jolla, CA, USA). For short hairpin RNA (shRNA), 3 optimal targeting human PDE11A and 1 scrambled control were designed (Additional file 1: Table S2). Lentiviral particles were generated by co-transfecting the transfer vector (psh2-u6-egfp-puro containing shRNA or scramble, pCDH-CMV-MCS-EF1-GFP-T2A-Puro containing WT PDE11A, PDE11A p.Arg202His or PDE11A p.Leu756GIn) and the packaging vectors into HEK293T cells.

\section{Cell culture and transfection}

HEK293 cells stably transfected with human WT APP695 (cells termed HEK293-APP 695 ) were propagated in DMEM with $10 \%$ fetal bovine serum (FBS) and $300 \mu \mathrm{g} / \mathrm{mL}$ G418 (Thermo Fisher Scientific, Waltham, MA, USA). HEK293 cells were cultured in DMEM with $10 \%$ FBS. Primary cortical neurons were isolated from E17-E18 C57BL/6J mouse embryos. Tissues were cut into tiny pieces and digested in $0.125 \%$ trypsin (Thermo Fisher Scientific) supplemented with DNase I ( $100 \mathrm{mg} / \mathrm{mL}$; Sigma-Aldrich, Saint Louis, MO, USA) for 15 minutes at $37^{\circ} \mathrm{C}$. The cells were then dissociated into a single-cell suspension and filtered through cell strainers. The cell pellet was resuspended, seeded onto 6-well plates-coated with poly-D-lysine (Sigma-Aldrich) and cultured in neurobasal medium supplemented with $2 \%$ B27, L-glutamine (2 mM), and 1\% penicillin/streptomycin (Thermo Fisher Scientific). Transfection was performed using Lipofectamine 3000 Reagent (Thermo Fisher Scientific).

\section{Real-time quantitative PCR}

Total RNA was purified from freshly harvested cells using an RNeasy Mini Kit (Thermo Fisher Scientific) and TRIzol (Thermo Fisher Scientific). Reverse transcription was carried out using a SuperScript First-Strand Synthesis Kit (Thermo Fisher Scientific), and total mRNA was measured by real-time quantitative polymerase chain reaction (PCR). Diluted cDNA templates, upstream and downstream primers and SYBR Green I Master mix (Thermo Fisher Scientific) were mixed in a $20-\mu \mathrm{L}$ volume, and reactions were performed using the StepOne Plus real-time PCR platform. Human GAPDH (forward, 5'-

ACAGCCTCAAGATCATCAGCAAT-3'; reverse, 5'- GATGGCATGGACTGTGGTCAT-3') was used as the internal reference gene, and relative expression levels of the PDE11A gene were calculated using the $2^{-\triangle \triangle C t}$ method. PDE11A q-PCR primers were as follows: forward, 5'- CTGGAGTGGATTGATAGCATCTG-3'; reverse, 5'CAGTCGTTTTTGgTGTAGCTCTT-3'.

\section{Western blotting}

Briefly, cells were lysed in RIPA buffer containing protease inhibitor cocktails and phosphatase inhibitors. The cell lysate was pelleted by centrifugation, and the protein concentration was measured by the BCA assay. Samples were separated by $10 \%$ Tris-glycine SDS-PAGE and then electroblotted onto a PVDF membrane. After blocking in 5\% nonfat dry milk, the membrane was probed with appropriate primary antibodies (listed in Additional file 1: Table S3) overnight at $4^{\circ} \mathrm{C}$. The next day, the membrane was washed with Tris-buffered saline containing Tween-20 (TBS-T) and incubated with horseradish peroxidase-linked secondary antibodies (1:5000; Santa Cruz Biotechnology) for $1 \mathrm{~h}$ at room temperature. After washing, the proteins were visualized by chemiluminescence.

\section{ELISA}

The concentrations of secreted $A \beta 40$ and $A \beta 42$ in conditioned medium were quantified using commercial enzyme-linked immunosorbent assay kits (IBL International, Hamburg, Germany). Levels of CAMP in cell lysates were determined using a commercially available assay kit (R\&D Systems, Minneapolis, MN, USA) according to the protocol provided by the manufacturer.

\section{Statistical analysis}


Quantification data were obtained from three independent repeats. Two-tailed unpaired Student's t test or one-way analysis of variance was performed using the SPSS 21.0 software package (SPSS, Chicago, IL, USA). To determine if the candidate risk gene is enriched in AD, a Fisher's exact test on the mutation count data was performed. A $p$ value less than 0.05 was used to indicate a statistically significant difference.

\section{Results}

\section{Identification of PDE11A variants}

We sequenced and analyzed the whole exome of 270 individuals (the pipeline is shown in Fig. 1). The cohort consisted of 215 EOAD cases and 55 unrelated control samples. About 80,000 variants per sample passed our quality control filters. For investigation of novel genes, patients carrying known EOAD risk genes (TREM2, VPS35, SORL1, MARK4, RUFY1 and TCIRG1) or genes associated with late-onset AD (ABCA7, ADAM17, IGHG3, PLD3, UNC5C, BIN1, CD2AP, $C L U, C R 1, E P H A 1, M S 4 A 4 A, P L C G 2, A B / 3, A K A P 9$ and ZNF655) were excluded ( $\mathrm{n}=13$ ). We then selected only mutations rare in the population (< $0.01 \% \mathrm{MAF})$ and coding mutations, lowering the count to 317 . Next, we excluded synonymous variants and used in silico analysis to restrict our findings to those predicted as damaging for the protein, revealing 32 variants. To further narrow the search for variants of interest, we used data from OMIM, MGI, GO, KEGG, ACGM and UKBiobank PheWeb to perform a systems-level analysis of the 32 mutated genes (Additional file 1: Table S4).

Among them, PDE11A met all criteria. We identified two variants in the PDE11A gene (NM_016953: rs752822096: c.605G>A: p.Arg202His and NM_016953: rs201572288: c.2267T>A: p.Leu756GIn). PDE11A is a dominant gene located on an autosome. A strong association was shown $\left(p\right.$-value $\left.=1.0 \times 10^{-13}\right)$ between PDE11A and AD using the UKBiobank PheWeb tool. Based on genetic databases, these two variants are rare in the East Asian population (p.Arg202His, gnomAD exomes_EAS: 0.0000; p.Leu756GIn, gnomAD exomes_EAS: 0.00431). Confirmation by Sanger sequencing is shown in Figure 2a. The p.Arg202His and p.Leu756Gln variants are predicted by eleven bioinformatics tools, including Polyphen2 HDIV, Polyphen2 HVAR, SIFT, LRT, PROVEAN, MutationTaster, DANN, VEST3, fathmm-MKL, CADD and M-CAP, to be damaging to the protein and by three algorithms (GERP, phastCons, phyloP) to be conserved (Table 1). The p.Arg202His and p.Leu756GIn variants are likely pathogenic and of uncertain significance, respectively, according to the American College of Medical Genetics and Genomics guidelines [19]. Subsequent Sanger sequencing analysis was performed in an expanded cohort of individuals ( $N=4962: 513$ EOAD, 4449 controls). We identified one p.Arg202His carriers in EOAD patients but none in normal controls, and six p.Leu756Gln carriers in EOAD patients and sixteen carriers in normal controls. Combined these two-stage sequencing, we found the two variants were significantly enriched in $A D$ compared to control respectively (p.Arg202His, 0.0028 versus $0, p=0.019$; p.Leu756GIn, 0.0097 versus $0.0036, p=0.032$; Fisher's exact test).

\section{PDE11A variants and clinical features}

The PDE11A p.Arg202His variant was detected in a 54-year-old female patient who had visited our hospital complaining of progressive memory decline over the past 4 years. She presented with amnesia as well as executive function and orientation deficits. She scored 9/30 on the MMSE and 8/30 on the Montreal Cognitive Assessment (MoCA), which was below the recommended cutoff values of 22 and 24, respectively. She had a CDR score of 3 and only remembered one word from the WHO-UCLA Delayed Recall Memory Test. MRI revealed moderate cerebral atrophy, especially in the hippocampus (MTA=4). Her APOE genotype was $\varepsilon 3 / \varepsilon 3$. Her parents and siblings were cognitively normal without complaints. Both her parents were deceased, and no DNA was available.

PDE11A p.Leu756Gln was found in a male patient who presented episodic memory decline at the age of 52 years. He had progressive difficulties in understanding and orientation, and he developed motor aphasia and personality changes in subsequent years. MRI showed atrophy of the temporoparietal lobe. The patient's APOE genotype was $\varepsilon 4 / \varepsilon 3$. He denied a family history of dementia.

\section{Functional annotation of rare $P D E 11 A$ variants}

PDE11A p.Arg202His is uniquely present in the PDE11A4 isoform. The PDE11A protein sequence in which the two rare variants are located is highly conserved amino acids across different species (Fig. 2b), and their GERP scores are 4.34 and 5.57 respectively, implicating potential interference of important protein biological functions by the mutations. PDE11A p.Arg202His and p.Leu756Gln are predicted by Poly-Phen2 and SIFT to be damaging or possibly damaging (Table 1). As depicted in the schematic diagram of full-length PDE11A in Figure 2C, the two variants are located near or in functional domains, including the cGMP-specific phosphodiesterase, adenylyl cyclase and FhIA (GAF) and catalytic domains, suggesting a potential functional impact of these variants on the PDE11A protein.

Global conformations of the p.Arg202His and p.Leu756Gln variants changed significantly from wild-type human PDE11A in three-dimensional (3D) homology models (Fig. 2d-f). Specifically, the 3D model predicts that p.Arg202His abolishes critical hydrogen bonds with surrounding amino acids; in contrast, p.Leu756GIn leads to a new hydrogen bonded network, which affects the helical structure. Taken together, the model predicts that both p.Arg202His and p.Leu756GIn variants identified in patients with AD may impair PDE11A function.

\section{PDE11A expression in AD brain tissues}

\section{Table 1. Pathogenicity Prediction of PDE11A Variants}




\begin{tabular}{|c|c|c|c|c|c|c|c|c|c|c|}
\hline Variant & SIFT & $\begin{array}{l}\text { PolyPhen2 } \\
\text { HDIV }\end{array}$ & $\begin{array}{l}\text { Polyphen2 } \\
\text { HVAR }\end{array}$ & LRT & MutationTaster & DANN & CADD & M-CAP & PROVEAN & VEST3 \\
\hline $\begin{array}{l}\text { c.G605A } \\
\text { (p.Arg202His) }\end{array}$ & $\begin{array}{l}\text { Damaging } \\
(0.028)\end{array}$ & $\begin{array}{l}\text { Probably } \\
\text { damaging } \\
(0.997)\end{array}$ & $\begin{array}{l}\text { Possibly } \\
\text { damaging } \\
(0.792)\end{array}$ & $\begin{array}{l}\text { Deleterious } \\
(0.000071)\end{array}$ & $\begin{array}{l}\text { Disease- } \\
\text { causing (1) }\end{array}$ & 0.998 & 6.96 & $\begin{array}{l}\text { Damaging } \\
(0.027)\end{array}$ & Damaging(-3.92) & 0.821 \\
\hline $\begin{array}{l}\text { c.T2267A } \\
\text { (p.Leu756GIn) }\end{array}$ & $\begin{array}{l}\text { Damaging } \\
(0.001)\end{array}$ & $\begin{array}{l}\text { Probably } \\
\text { damaging } \\
(0.996)\end{array}$ & $\begin{array}{l}\text { Probably } \\
\text { damaging } \\
(0.943)\end{array}$ & $\begin{array}{l}\text { Deleterious } \\
\text { (0) }\end{array}$ & $\begin{array}{l}\text { Disease- } \\
\text { causing (1) }\end{array}$ & 0.995 & 6.12 & $\begin{array}{l}\text { Damaging } \\
(0.174)\end{array}$ & $\begin{array}{l}\text { Damaging } \\
(-4.71)\end{array}$ & 0.952 \\
\hline
\end{tabular}

The PDE11A gene is expressed in several regions of the mouse hippocampus, including the CA1, the subiculum, and the amygdalohippocampal area [20]. Moreover, Pde11a-knockout mice exhibit enlarged lateral ventricles and abnormal social investigation [20]. PDE11A is also reported to be involved in the regulation of cGMP-mediated signaling. These results suggest that PDE11A has an important role in the brain, with a possible role in central nervous system disorders.

We assessed PDE11A expression in single-nuclei RNA sequencing data from AD cases and control brain samples[18] and found the PDE11A gene to be expressed in almost all types of cells, including neurons, astrocytes, and microglia. (Fig. 3a-b).

To characterize the involvement of PDE11A in AD, we analyzed the PDE11A protein in fresh frozen postmortem brain tissues from cognitively normal healthy controls $(n=6)$ and patients with $A D(n=6)$. All cases were matched for age and sex. This analysis revealed significantly decreased levels of PDE11A in those with $A D$ relative to healthy controls (Fig. 3c-d).

\section{Effects of PDE11A variants on $A \beta$ homeostasis}

To further confirm the pathogenesis of PDE11A variants, we performed in vitro studies to test the effect on A homeostasis. Lower levels of the PDE11A protein was observed in AD patients. Therefore, we used PDE11A shRNA to knockdown (KD) PDE11A levels in cell models. A markedly lower PDE11A mRNA and protein levels were obtained in HEK cells (Additional file 2: Figure S1A-C).

HEK293-APP 695 cells were transfected with the PDE11A plasmid (PDE11A WT, p.Arg202His, p.Leu756GIn, scramble or shRNA) showed no significant differences in secreted $A \beta_{40}$ or $A \beta_{42}$ levels or the $A \beta_{42} / A \beta_{40}$ ratio (Additional file 2: Figure S2A-C). PDE11A variants or KD did not change the levels of $A P P$ or BACE-1(Additional file 2: Figure S3A-C). These results suggest that PDE11A may not affect A $\beta$ homeostasis.

\section{PDE11A variants affect Tau phosphorylation}

To understand the influence of PDE11A variants on Tau phosphorylation, lentiviruses with human MAPT and PDE11A (WT PDE11A, PDE11A p.Arg202His, PDE11A p.Leu756GIn, shRNA or scramble) were used to transduce primary neurons simultaneously. Effects on Tau phosphorylation levels in transduced primary neurons were assessed by immunoblotting. A significant reduction in Tau phosphorylation was detected at multiple sites, including T181, S404, S202/T205, S416, S214, and S396, in WT PDE11A-expressing neurons compared to neurons infected with mock lentivirus (Fig. 4). Moreover, both variants notably increased Tau phosphorylation at multiple sites compared with the WT (Fig. 4a-b). PDE11A shRNA treatment reduced PDE11A mRNA and protein levels by $60 \%$ and significantly increased Tau phosphorylation at multiple sites compared with scramble shRNA treatment (Fig. 4a-c). These results suggest that both mutations could be a loss-of-function.

The protein level of GSK-3 $\beta$, a major kinase involved in Tau phosphorylation, and its inactive form p-Ser9-GSK3 $\beta$ were not significantly altered in any of the groups (Additional file 2: Figure S4A-C). These data suggest that PDE11A variants likely affect Tau phosphorylation independent of GSK-3 $\beta$ signaling.

\section{PDE11A variantsexhibit alterations in cAMP/PKA signaling}

The cAMP/PKA/CREB signaling plays an important role in AD. However, the link with PDE11A is unknown. To further clarify the underlying mechanisms, we tested the effects of PDE11A on CAMP/PKA/CREB signaling. Transduction of WT PDE11A (with MAPT) in primary neurons decreased cAMP levels compared to the mock group (with MAPT). The p.Arg202His and p.Leu756GIn variants increased cAMP levels relative to WT PDE11A (Fig. 5a-b). These results suggest that the p.Arg202His and p.Leu756Gln variants reduced the ability of PDE11A to degrade cAMP. Total PKA and p(Thr197)-PKA levels, as well as the ratio of phosphorylated CREB ( $p$-CREB) to CREB, were also increased in variants PDE11A-expressing neurons compared to WT PDE11A-expressing neurons (Fig. 5c-d). Similar results were obtained in PDE11A shRNA-treated neurons compared to scramble shRNA-treated neurons (Fig. 5c and 5e).

In addition, pretreating the neurons with the PKA inhibitor (H89) reduced PDE11A p.Arg202His and p.Leu756GIn variant-induced Tau phosphorylation (Fig. 6ac). H89 pretreatment also decrease p-PKA and p-CREB/CREB levels (Fig. $6 \mathrm{~d}-\mathrm{h}$ ). Furthermore, these results were recapitulated using PDE11A shRNA. Therefore, these data demonstrate that the PDE11A variants affect the cAMP/PKA pathway, which is associated with increased Tau phosphorylation through a loss-offunction mechanism.

\section{Discussion}

In the present study, we report PDE11A as a novel candidate risk gene for EOAD. Our results showed that PDE11A variants did not affect amyloid production but cause dysregulation of CAMP/PKA signaling and increase Tau phosphorylation. These findings potentially improve our understanding of the molecular mechanisms of EOAD and reveal new AD target pathways. 
We selected 215 cases of very early-onset $A D$ (EOAD, with age of onset before 55 years) to detect new AD risk genes. EOAD has higher heritability than lateonset $A D$ [21] and absence of known causal or risk $A D$ gene mutations, sequencing EOAD increases the chances of discovering new $A D$ genes. Using a systematic analysis of WES data, we identified PDE11A as a novel candidate risk gene for EOAD. Combined with the subsequent direct sequencing of the two variants in 4962 individuals, it revealed the two variants were significantly enriched in AD. The human PDE11A gene is located at $2 q 31.2$ and consists of 23 exons [22]. Four transcript isoforms designated PDE11A1-4, which result from different transcription initiation sites and alternative splicing, encode different PDE11A isoforms with unique N-terminal domains. Germline mutations, expression changes, and functional alterations in PDE11A have been linked to brain function, tumorigenesis, and inflammation [20, 23, 24]. For example, PDE11A is required for intact brain function across the lifespan [25], and studies have suggested that variations in PDE11A may be associated with bipolar disorder, depression and other psychiatric diseases [26, 27]. Moreover, PDE11A negatively regulates the efficacy of lithium in treating bipolar disorder [28]. Expression of PDE11A4 mRNA in the brain tends to occur in CA1 neurons and in the subiculum in ventral hippocampal (VHIPP) formation. Atrophy of VHIPP has been observed in patients with AD or mild cognitive impairment [29, 30]. Importantly, the extent of anatomical and functional deficits in the VHIPP appears to correlate with the severity of clinical impairment in patients with AD [30, 31]. In addition, PDE11A4 can directly influence synaptic plasticity [32]. PDE11A4 also plays a role in systems consolidation, which has relevance for cognitive deficits [33]. Phosphodiesterases (PDEs) have been linked to mental processes of emotions, learning and memory, with implications in mood and cognitive disorders, including Huntington disease, Parkinson's disease and AD [34-37]. This evidence suggests that PDE11A plays a role in brain function and may also be involved in $A D$.

In our study, we identified two rare nonsynonymous variants p.Arg202His and p.Leu756GIn in PDE11A in EOAD patients. The p.Arg202His variant is only present in the PDE11A4 isoform, whereas the p.Leu756GIn variant is present in all PDE11A isoforms. PDE11A isoforms are expressed in a tissue-specific manner [24]. In a previous study, Kelly et al. found that PDE11A4 is highly expressed in hippocampal areas but not in any of the other tissues examined. PDE11A4 is the only PDE with restricted expression in hippocampal areas, which are affected in AD [38]. Both variants identified in this study affect PDE11A4 isoforms though a loss of function mechanism. Both variants are highly conserved evolutionarily and located near or in an important PDE11A4 domain. In addition, both mutations disrupt hydrogen bonding and dramatically alter protein coformation. Furthermore, both variants are predicted to be damaging by eleven in silico tools and are considered conserved by all four algorithms applied. Taken together, these findings suggest that important biological functions can be attributed to these variants. Moreover, using bulk RNA-Seq data derived from 1536 individuals [39], we found that the PDE11A gene is significantly associated with neuronal proportion in cortical tissues. Neuronal loss in the cerebral cortex is one of the main characteristic pathological changes of $A D$. We also detected significantly decreased protein levels of PDE11A in brain tissues from AD patients. It appears that these variants may affect PDE11A function in the brain and facilitate disease onset.

We conducted cellular and biochemical studies and found that the PDE11A variants increase Tau phosphorylation at multiple sites. PDE11A shRNA also induces significant Tau hyperphosphorylation, whereas overexpression of PDE11A markedly decreases Tau phosphorylation. These findings suggest a putative role for $P D E 11 A$ and variants in tau phosphorylation. In contrast, the conditioned medium of cells transduced with lentivirus carrying the $\mathrm{p}$.Arg202His or p.Leu756GIn variant or PDE11A shRNA showed no significant differences in $A \beta_{42}$ or $A \beta_{40}$ levels or $A \beta_{42}$ to $A \beta_{40}$ ratio, indicating that the variants may not affect $A \beta$ peptides homeostasis. GSK-3 $\beta$ signaling affects Tau hyperphosphorylation. However, $P D E 11 A$ variants or shRNA groups did not affect GSK-3 $\beta$

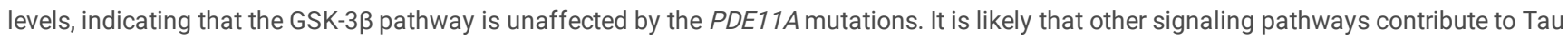
hyperphosphorylation. The underlying mechanisms connecting PDE11A and Tau needs to be further evaluated.

Our data demonstrate that PDE11A affects Tau phosphorylation through the CAMP/PKA pathway. PDEs hydrolyze cAMP and are key enzymes regulating intracellular cAMP levels. CAMP is known as a crucial second messenger that participates in transducing signals in many types of cells. cAMP activates PKA, which phosphorylates and activates CREB. Activated CREB binds to CREs in target genes to regulate their expression. CAMP/PKA signaling pathway dysregulation has been reported in $A D$ patients, and the pathway plays a crucial role in AD pathogenesis [40]. Therefore, we reasoned that $P D E 11 A$ variants might affect AD-relevant phentypes through cAMP/PKA signaling. Our results showed higher cAMP levels in lysates from cells transduced with $P D E 11 A$ p.Arg202His, p.Leu756GIn or shRNA compared with those expressing WT PDE11A or scramble shRNA. We also found that PDE11A p.Arg202His or p.Leu756Gln variant led to elevated PKA, p-PKA and p-CREB levels. Increases in cAMP levels and PKA activity have been observed in the cerebral microvessels of $A D$ patients [41], and elevated levels of cAMP in the CSF of AD patients have been reported [42]. Van et al. [43] documented an increase p-PKA levels in the temporal cortex of early-stage AD patients. Expression of PKA and p-CREB is decreased in AD patients and in vitro models [44, 45], suggesting that PKA and pCREB levels may vary on different tissues at different stages of disease progression.

PKA phosphorylates Tau, and it is important for the formation of NFTs [46, 47]. Martinez et al.[42] showed that cAMP levels correlate with levels of Tau protein in the CSF. In the present study, $\mathrm{H} 89$ (a specific PKA inhibitor) pretreatment suppressed PDE11A mutation-associated p-Tau formation, which further confirmed the role of the CAMP/PKA/CREB signaling pathway in PDE11A-mediated regulation of Tau phosphorylation. Carlyle et al.[48] reported an age-related increase in cAMP-dependent PKA-mediated phosphorylation of Tau as a risk factor for aged cortex degeneration. PKA activation induces Tau hyperphosphorylation and spatial memory deficits, which are reversed by the PKA inhibitor H89 inhibits [47]. Ikezu et al. [49] identified two rare variants in AKAP9 in African American AD patients. AKAP9 mutations had no effect on A $\beta$ but significantly increased Tau phosphorylation in cells treated with a PDE4 inhibitor [50]. PKA enhances Tau phosphorylation by other Tau kinases [51]. Thus, the effect of PKA on AD-associated Tau phosphorylation may be either direct or indirect, and the exact mechanism has yet to be elucidated.

\section{Limitations}

First, the individuals carrying the $P D E 11 A$ variants did not report AD family history. Due to absence of DNA samples from relatives, we could not determine whether these PDE11A mutations are de novo variants. Second, an independent replication of the association of PDE11A variants in different populations would have been confirmatory of our genetic findings. Third, our results highlight the need to investigate the role of PDE11A in AD using in vivo models in 
future research. Such in vivo studies will provide evidence of PDE11A effects on cognition and confirm the mechanism through which PDE11A is involved in AD.

\section{Conclusion}

We report for the first time two novel rare PDE11A variants in four individuals with EOAD. These PDE11A variants enhance both cAMP/PKA signaling and Tau phosphorylation. PDE11A could be a novel candidate genetic predisposing factor in AD.

\section{Abbreviations}

AD: Alzheimer's disease; EOAD: early-onset AD; LOAD: Late-onset Alzheimer's disease; WES: whole-exome sequencing; PDE11A: phosphodiesterase 11A; shRNA: specific short hairpin RNA; PKA: protein kinase A; CREB: CAMP response element-binding protein; AB: amyloid- $\beta$; $p$-Tau: phosphorylated tau; AAO: age at onset; GWAS: Genome-wide association studies; APP. amyloid precursor protein; MRI: magnetic resonance imaging; MMSE: Mini-Mental State Examination; MoCA: Montreal Cognitive Assessment; PCR: polymerase chain reaction; VHIPP: subiculum in ventral hippocampal.

\section{Declarations}

\section{Acknowledgements}

We thank the many institutions and their staff that provided support for this study and who were involved in this collaboration. We sincerely thank the patients and their relatives for participation.

\section{Authors' contributions}

Qin Wei designed the project, performed the experiments, wrote and edited the manuscript; Aihong Zhou and Xiumei Zuo examined the patients; Longfei Jia, Cuibai Wei, Yi Tang and Fen Wang interpreted the data; Fangyu Li analysed and interpreted data. Qi Wang conducted sanger sequence; Ying Li extracted DNA samples; Yiping Wei helped to culture primary cortical neurons; Lina Zhao and Hongmei Jin performed cognitive tests on participants; Carlos Cruchaga interpreted the data and edited the manuscript; Bruno A. Benitez detected PDE11A protein expression in brain tissues and edited the manuscript; Jianping Jia designed the study and edited the manuscript. All authors read and approved the final manuscript.

\section{Funding}

This study was supported by the Key Project of the National Natural Science Foundation of China (81530036); Beijing Natural Science Foundation (7192077); National Key R\&D Program of China (2017YFC1310100); the National Key Scientific Instrument and Equipment Development Project (31627803); Beijing Municipal Science \& Technology Commission (Z181100001718110); Mission Program of Beijing Municipal Administration of Hospitals (SML20150801); Beijing Scholars Program; Project for Outstanding Doctor with Combined Ability of Western and Chinese Medicine; Beijing Municipal Commission of Health and Family Planning(PXM2019_026283_000003); and grants from the National Institutes of Health (R01AG044546, P01AG003991, RF1AG053303, R01AG058501, U01AG058922, RF1AG058501 and R01AG064614).

\section{Availability of data and materials}

The data generated and analyzed in this study are available from the corresponding author on reasonable request.

\section{Ethics approval}

The study protocol was approved and monitored by the Ethics Committee of Xuanwu Hospital. Signed informed consent was provided by all the patients and control subjects. The recruited fresh frozen postmortem parietal lobe tissues were approved by the institutional review board of Washington University. Written informed consent for brain autopsy was obtained from all participants or their legal representatives.

\section{Consent for publication}

All authors have approved of the manuscript and agree with its submission.

\section{Competing interests}

All authors except Carlos Cruchaga report no disclosures. Carlos Cruchaga receives research support from: Biogen, EISAl, Alector and Parabon. The funders of the study had no role in the collection, analysis, or interpretation of data; in the writing of the report; or in the decision to submit the paper for publication. Carlos Cruchaga is a member of the advisory board of Vivid genetics, Halia Therapeutics and ADx Healthcare.

\section{References}

1. Collaborators GBDD. Global, regional, and national burden of Alzheimer's disease and other dementias, 1990-2016: a systematic analysis for the Global Burden of Disease Study 2016. Lancet Neurol. 2019;18:88-106.

2. Brookmeyer R, Johnson E, Ziegler-Graham K, Arrighi HM. Forecasting the global burden of Alzheimer's disease. Alzheimers Dement. 2007;3:186-191. 
3. Hyman BT, Phelps CH, Beach TG, Bigio EH, Cairns NJ, Carrillo MC, Dickson DW, Duyckaerts C, Frosch MP, Masliah E, et al. National Institute on AgingAlzheimer's Association guidelines for the neuropathologic assessment of Alzheimer's disease. Alzheimer's \& dementia : the journal of the Alzheimer's Association. 2012;8:1-13.

4. Sims R, Hill M, Williams J. The multiplex model of the genetics of Alzheimer's disease. Nat Neurosci. 2020;23:311-322.

5. Barber IS, Braae A, Clement N, Patel T, Guetta-Baranes T, Brookes K, Medway C, Chappell S, Guerreiro R, Bras J, et al. Mutation analysis of sporadic earlyonset Alzheimer's disease using the NeuroX array. Neurobiol Aging. 2017;49:215 e211-215 e218.

6. Lanoiselee HM, Nicolas G, Wallon D, Rovelet-Lecrux A, Lacour M, Rousseau S, Richard AC, Pasquier F, Rollin-Sillaire A, Martinaud O, et al. APP, PSEN1, and PSEN2 mutations in early-onset Alzheimer disease: A genetic screening study of familial and sporadic cases. PLoS Med. 2017;14:e1002270.

7. Bis JC, Jian X, Kunkle BW, Chen Y, Hamilton-Nelson KL, Bush WS, Salerno WJ, Lancour D, Ma Y, Renton AE, et al. Whole exome sequencing study identifies novel rare and common Alzheimer's-Associated variants involved in immune response and transcriptional regulation. Mol Psychiatry. 2018.

8. Ma Y, Jun GR, Zhang X, Chung J, Naj AC, Chen Y, Bellenguez C, Hamilton-Nelson K, Martin ER, Kunkle BW, et al. Analysis of Whole-Exome Sequencing Data for Alzheimer Disease Stratified by APOE Genotype. JAMA Neurol. 2019.

9. Hartl D, May P, Gu W, Mayhaus M, Pichler S, Spaniol C, Glaab E, Bobbili DR, Antony P, Koegelsberger S, et al. A rare loss-of-function variant of ADAM17 is associated with late-onset familial Alzheimer disease. Mol Psychiatry. 2018.

10. Zhang X, Zhu C, Beecham G, Vardarajan BN, Ma Y, Lancour D, Farrell JJ, Chung J, Alzheimer's Disease Sequencing P, Mayeux R, et al. A rare missense variant of CASP7 is associated with familial late-onset Alzheimer's disease. Alzheimer's \& dementia : the journal of the Alzheimer's Association. 2019;15:441-452.

11. De Roeck A, Van Broeckhoven C, Sleegers K. The role of ABCA7 in Alzheimer's disease: evidence from genomics, transcriptomics and methylomics. Acta Neuropathol. 2019;138:201-220.

12. McKhann G, Drachman D, Folstein M, Katzman R, Price D, Stadlan EM. Clinical diagnosis of Alzheimer's disease: report of the NINCDS-ADRDA Work Group under the auspices of Department of Health and Human Services Task Force on Alzheimer's Disease. Neurology. 1984;34:939-944.

13. McKhann GM, Knopman DS, Chertkow H, Hyman BT, Jack CR, Jr., Kawas CH, Klunk WE, Koroshetz WJ, Manly JJ, Mayeux R, et al. The diagnosis of dementia due to Alzheimer's disease: recommendations from the National Institute on Aging-Alzheimer's Association workgroups on diagnostic guidelines for Alzheimer's disease. Alzheimer's \& dementia : the journal of the Alzheimer's Association. 2011;7:263-269.

14. Mitchell AL, Attwood TK, Babbitt PC, Blum M, Bork P, Bridge A, Brown SD, Chang HY, El-Gebali S, Fraser MI, et al. InterPro in 2019: improving coverage, classification and access to protein sequence annotations. Nucleic Acids Res. 2019;47:D351-D360.

15. Webb B, Sali A. Comparative Protein Structure Modeling Using MODELLER. Curr Protoc Bioinformatics. 2016;54:5 6 1-5 637.

16. Janson G, Zhang C, Prado MG, Paiardini A. PyMod 2.0: improvements in protein sequence-structure analysis and homology modeling within PyMOL. Bioinformatics. 2017;33:444-446.

17. Wang LP, McKiernan KA, Gomes J, Beauchamp KA, Head-Gordon T, Rice JE, Swope WC, Martinez TJ, Pande VS. Building a More Predictive Protein Force Field: A Systematic and Reproducible Route to AMBER-FB15. J Phys Chem B. 2017;121:4023-4039.

18. Grubman A, Chew G, Ouyang JF, Sun G, Choo XY, McLean C, Simmons RK, Buckberry S, Vargas-Landin DB, Poppe D, et al. A single-cell atlas of entorhinal cortex from individuals with Alzheimer's disease reveals cell-type-specific gene expression regulation. Nat Neurosci. 2019;22:2087-2097.

19. Richards S, Aziz N, Bale S, Bick D, Das S, Gastier-Foster J, Grody WW, Hegde M, Lyon E, Spector E, et al. Standards and guidelines for the interpretation of sequence variants: a joint consensus recommendation of the American College of Medical Genetics and Genomics and the Association for Molecular Pathology. Genet Med. 2015;17:405-424.

20. Kelly MP, Logue SF, Brennan J, Day JP, Lakkaraju S, Jiang L, Zhong X, Tam M, Sukoff Rizzo SJ, Platt BJ, et al. Phosphodiesterase 11A in brain is enriched in ventral hippocampus and deletion causes psychiatric disease-related phenotypes. Proc Natl Acad Sci U S A. 2010;107:8457-8462.

21. Wingo TS, Lah JJ, Levey Al, Cutler DJ. Autosomal recessive causes likely in early-onset Alzheimer disease. Arch Neurol. 2012;69:59-64.

22. Fawcett L, Baxendale R, Stacey P, McGrouther C, Harrow I, Soderling S, Hetman J, Beavo JA, Phillips SC. Molecular cloning and characterization of a distinct human phosphodiesterase gene family: PDE11A. Proc Natl Acad Sci U S A. 2000;97:3702-3707.

23. Vezzosi D, Libe R, Baudry C, Rizk-Rabin M, Horvath A, Levy I, Rene-Corail F, Ragazzon B, Stratakis CA, Vandecasteele G, Bertherat J. Phosphodiesterase $11 \mathrm{~A}$ (PDE11A) gene defects in patients with acth-independent macronodular adrenal hyperplasia (AIMAH): functional variants may contribute to genetic susceptibility of bilateral adrenal tumors. J Clin Endocrinol Metab. 2012;97:E2063-2069.

24. Kelly MP. Does phosphodiesterase 11A (PDE11A) hold promise as a future therapeutic target? Curr Pharm Des. 2015;21:389-416.

25. Kelly MP, Adamowicz W, Bove S, Hartman AJ, Mariga A, Pathak G, Reinhart V, Romegialli A, Kleiman RJ. Select 3',5'-cyclic nucleotide phosphodiesterases exhibit altered expression in the aged rodent brain. Cell Signal. 2014;26:383-397.

26. Wong ML, Whelan F, Deloukas P, Whittaker P, Delgado M, Cantor RM, McCann SM, Licinio J. Phosphodiesterase genes are associated with susceptibility to major depression and antidepressant treatment response. Proc Natl Acad Sci U S A. 2006;103:15124-15129.

27. Coon H, Darlington T, Pimentel R, Smith KR, Huff CD, Hu H, Jerominski L, Hansen J, Klein M, Callor WB, et al. Genetic risk factors in two Utah pedigrees at high risk for suicide. Transl Psychiatry. 2013;3:e325.

28. Pathak G, Agostino MJ, Bishara K, Capell WR, Fisher JL, Hegde S, Ibrahim BA, Pilarzyk K, Sabin C, Tuczkewycz T, et al. PDE11A negatively regulates lithium responsivity. Mol Psychiatry. 2017;22:1714-1724.

29. Lee P, Ryoo H, Park J, Jeong Y, Alzheimer's Disease Neuroimaging I. Morphological and Microstructural Changes of the Hippocampus in Early MCl: A Study Utilizing the Alzheimer's Disease Neuroimaging Initiative Database. J Clin Neurol. 2017;13:144-154. 
30. Nie X, Sun Y, Wan S, Zhao H, Liu R, Li X, Wu S, Nedelska Z, Hort J, Qing Z, et al. Subregional Structural Alterations in Hippocampus and Nucleus Accumbens Correlate with the Clinical Impairment in Patients with Alzheimer's Disease Clinical Spectrum: Parallel Combining Volume and Vertex-Based Approach. Front Neurol. 2017;8:399.

31. Yakushev I, Gerhard A, Muller MJ, Lorscheider M, Buchholz HG, Schermuly I, Weibrich C, Hammers A, Stoeter P, Schreckenberger M, Fellgiebel A. Relationships between hippocampal microstructure, metabolism, and function in early Alzheimer's disease. Brain Struct Funct. 2011;216:219-226.

32. Hegde S, Capell WR, Ibrahim BA, Klett J, Patel NS, Sougiannis AT, Kelly MP. Phosphodiesterase 11A (PDE11A), Enriched in Ventral Hippocampus Neurons, is Required for Consolidation of Social but not Nonsocial Memories in Mice. Neuropsychopharmacology. 2016;41:2920-2931.

33. Pilarzyk K, Klett J, Pena EA, Porcher L, Smith AJ, Kelly MP. Loss of Function of Phosphodiesterase 11A4 Shows that Recent and Remote Long-Term Memories Can Be Uncoupled. Curr Biol. 2019;29:2307-2321 e2305.

34. Tibbo AJ, Tejeda GS, Baillie GS. Understanding PDE4's function in Alzheimer's disease; a target for novel therapeutic approaches. Biochem Soc Trans. 2019;47:1557-1565.

35. Cheng J, Liao Y, Dong Y, Hu H, Yang N, Kong X, Li S, Li X, Guo J, Qin L, et al. Microglial autophagy defect causes parkinson disease-like symptoms by accelerating inflammasome activation in mice. Autophagy. 2020:1-13.

36. Fazio P, Fitzer-Attas CJ, Mrzljak L, Bronzova J, Nag S, Warner JH, Landwehrmeyer B, Al-Tawil N, Halldin C, Forsberg A, et al. PET Molecular Imaging of Phosphodiesterase 10A: An Early Biomarker of Huntington's Disease Progression. Mov Disord. 2020;35:606-615.

37. Farmer R, Burbano SD, Patel NS, Sarmiento A, Smith AJ, Kelly MP. Phosphodiesterases PDE2A and PDE10A both change mRNA expression in the human brain with age, but only PDE2A changes in a region-specific manner with psychiatric disease. Cell Signal. 2020;70:109592.

38. Kelly MP. A Role for Phosphodiesterase 11A (PDE11A) in the Formation of Social Memories and the Stabilization of Mood. Adv Neurobiol. 2017;17:201230.

39. Li Z, Farias FHG, Dube U, Del-Aguila JL, Mihindukulasuriya KA, Fernandez MV, Ibanez L, Budde JP, Wang F, Lake AM, et al. The TMEM106B FTLDprotective variant, rs1990621, is also associated with increased neuronal proportion. Acta Neuropathol. 2020;139:45-61.

40. Masutomi H, Kawashima S, Kondo Y, Uchida Y, Jang B, Choi EK, Kim YS, Shimokado K, Ishigami A. Induction of peptidylarginine deiminase 2 and 3 by dibutyryl cAMP via cAMP-PKA signaling in human astrocytoma U-251MG cells. J Neurosci Res. 2017;95:1503-1512.

41. Martinez M, Hernandez Al, Hernanz A. Increased cAMP immunostaining in cerebral vessels in Alzheimer's disease. Brain Res. 2001;922:148-152.

42. Martinez M, Fernandez E, Frank A, Guaza C, de la Fuente M, Hernanz A. Increased cerebrospinal fluid cAMP levels in Alzheimer's disease. Brain Res. 1999;846:265-267.

43. van der Harg JM, Eggels L, Bangel FN, Ruigrok SR, Zwart R, Hoozemans JJM, la Fleur SE, Scheper W. Insulin deficiency results in reversible protein kinase A activation and tau phosphorylation. Neurobiol Dis. 2017;103:163-173.

44. Bonkale WL, Cowburn RF, Ohm TG, Bogdanovic N, Fastbom J. A quantitative autoradiographic study of [3H]cAMP binding to cytosolic and particulate protein kinase A in post-mortem brain staged for Alzheimer's disease neurofibrillary changes and amyloid deposits. Brain Res. 1999;818:383-396.

45. Du H, Guo L, Wu X, Sosunov AA, McKhann GM, Chen JX, Yan SS. Cyclophilin D deficiency rescues Abeta-impaired PKA/CREB signaling and alleviates synaptic degeneration. Biochim Biophys Acta. 2014;1842:2517-2527.

46. Kelly MP. Cyclic nucleotide signaling changes associated with normal aging and age-related diseases of the brain. Cell Signal. 2018;42:281-291.

47. Wang HH, Li Y, Li A, Yan F, Li ZL, Liu ZY, Zhang L, Zhang J, Dong WR, Zhang L. Forskolin Induces Hyperphosphorylation of Tau Accompanied by Cell Cycle Reactivation in Primary Hippocampal Neurons. Mol Neurobiol. 2018;55:696-706.

48. Carlyle BC, Nairn AC, Wang M, Yang Y, Jin LE, Simen AA, Ramos BP, Bordner KA, Craft GE, Davies P, et al. cAMP-PKA phosphorylation of tau confers risk for degeneration in aging association cortex. Proc Natl Acad Sci U S A. 2014;111:5036-5041.

49. Logue MW, Schu M, Vardarajan BN, Farrell J, Bennett DA, Buxbaum JD, Byrd GS, Ertekin-Taner N, Evans D, Foroud T, et al. Two rare AKAP9 variants are associated with Alzheimer's disease in African Americans. Alzheimer's \& dementia : the journal of the Alzheimer's Association. 2014;10:609-618 e611.

50. Ikezu T, Chen C, DeLeo AM, Zeldich E, Fallin MD, Kanaan NM, Lunetta KL, Abraham CR, Logue MW, Farrer LA. Tau Phosphorylation is Impacted by Rare AKAP9 Mutations Associated with Alzheimer Disease in African Americans. J Neuroimmune Pharmacol. 2018;13:254-264.

51. Liu F, Liang Z, Shi J, Yin D, El-Akkad E, Grundke-lqbal I, lqbal K, Gong CX. PKA modulates GSK-3beta- and cdk5-catalyzed phosphorylation of tau in siteand kinase-specific manners. FEBS Lett. 2006;580:6269-6274.

\section{Figures}




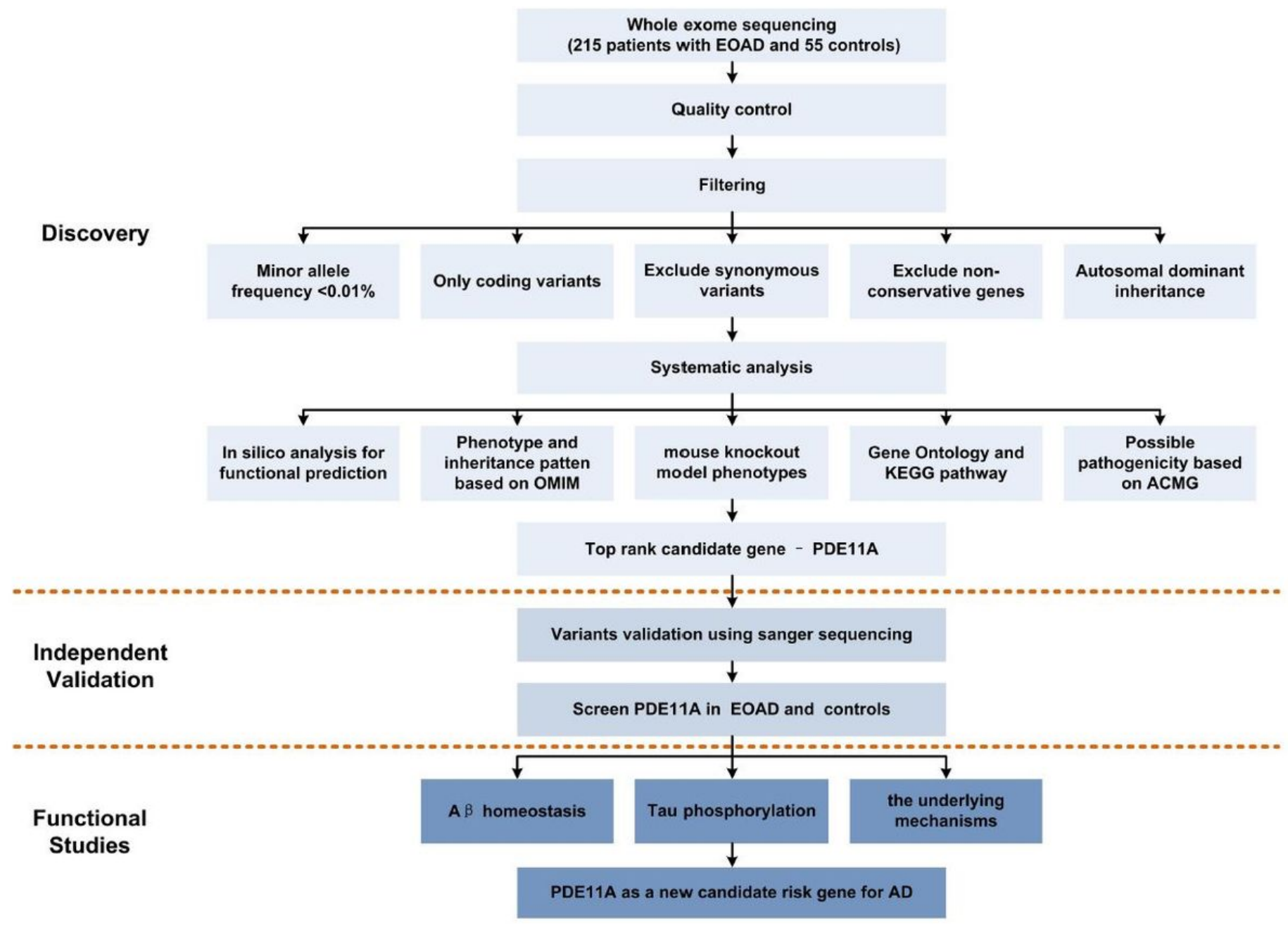

\section{Figure 1}

Workflow of the current study. WES was performed in 215 Chinese individuals with EOAD and 55 controls. Subsequent direct sequencing was performed in an independent cohort to validate the selected rare mutations. Then in vitro functional studies were performed to evaluate the role of candidate mutations in EOAD and the underlying mechanisms. 

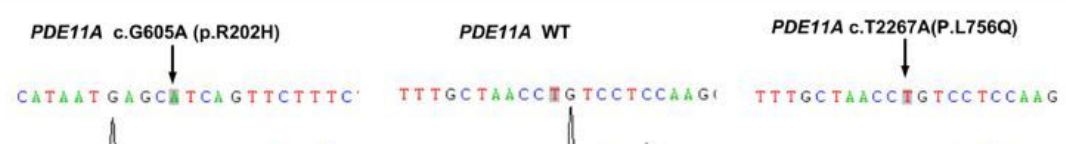

A
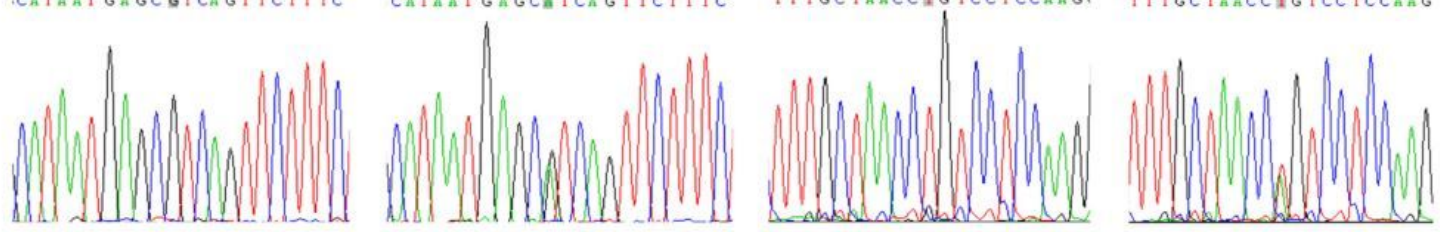

Homo sapiens.seq Rattus norvegicus.seg Mus_mus̄culus.seq

B Gallus_gallus.seq Cyprinus_carpio.seq Danio_rerio.seg Microtus ochrogaster.seq

R202H

SRVNL FRYFFTAILYKCHLKKHNERTFFLELVKLISNLID SRVNI PCYPFTAIIYKCHI KKHNGR FFFIELVKLISNLID SRVNLFCYFFTAICYKCHIKKHNERCFFLELVKLISNCID SRVSLFCYFFFALCYKRYLKEHNER EFFIELVKLISNLID SRVNIFCYFSTAVLYKYHLKEHNEREF FLEIVKLISNLID SRVNIFCYFSTAVLYKYYLKEHNEREF FLELVKLISNLID SRVNL FCYFFTAICYKCHLKKHNERCFFLELVKLISNCID SRVNIFCYFSTAVLFKYYLKEHNEREFFLELVKCISNCID

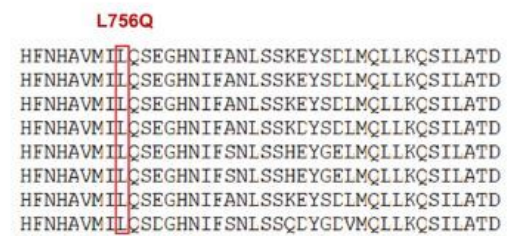

HFNHAVM IITCSEGHNIFANLSSKEYSCLMCLLLKÇSILATD TFNHAVMIII SEGHNIFANLSSKEYSCIMCLIKCSILATD FFNHAVMIIISEGHNIFANLSSKEYSDIMCLILCSILATD HFNHAVMII SEGHNIFSNISSHEYGEIMCLIKCQSILATD HFNHAVMIICSLGHNIF SNLSSCLYGLVMCLLLKÇSILATD

C

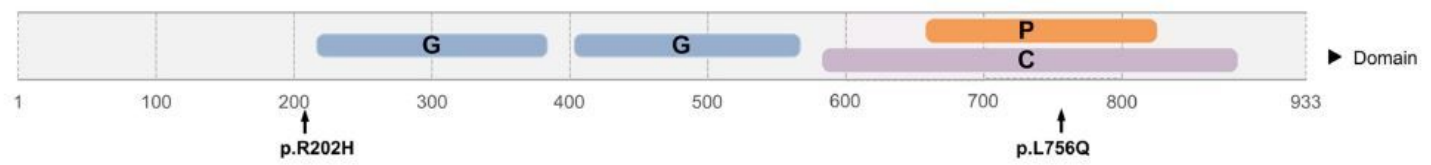

D

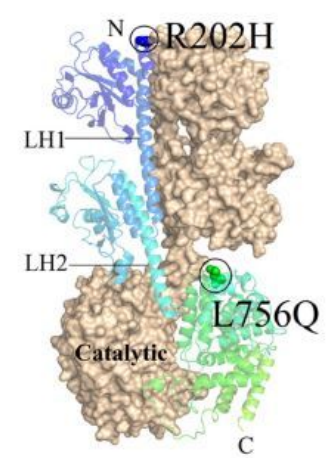

E

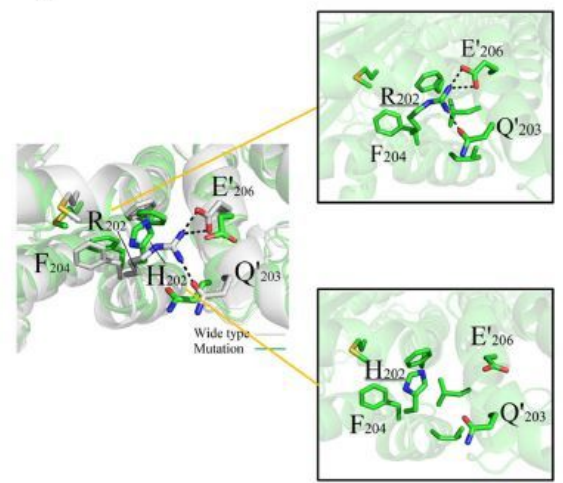

$\mathbf{F}$

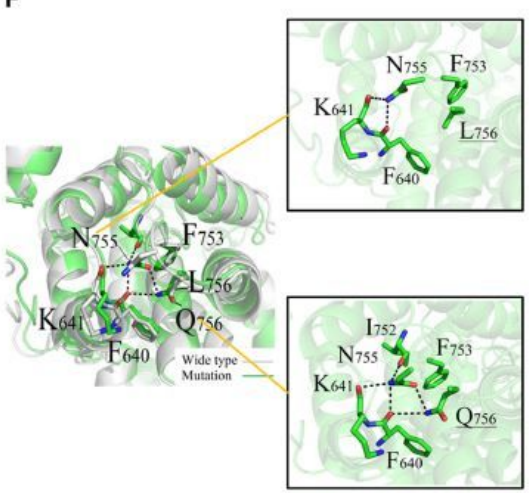

\section{Figure 2}

Functional annotation of PDE11A variants. (A) Sanger sequencing confirmation of PDE11A variants. (B) Protein homologs in different species were aligned. The p.Arg202His and p.Leu756Gln are conserved in all PDE11A orthologs. The variants are highlighted in the red boxes. (C) Predicted domain maps of PDE11A protein with the identified missense variants marked: G: GAF domain (aa 217-380 and aa402-568), P: HD/PDEase domain (aa 663-839), C: 3'5'-cyclic nucleotide phosphodiesterase, catalytic domain (aa 588-912). The p.Arg202His variant is located near the GAF domain, and p.Leu756Gln variant in the 3'5'cyclic nucleotide phosphodiesterase, catalytic domain. (D) Predicted three-dimensional structure of WT and mutant PDE11A protein. (E) Structure comparison between wild type PDE11A and R202H variant. Critical hydrogen bonds with surrounding amino acids were predicted to be eliminated. Dashed lines indicate hydrogen bonds. (F) Structure comparison between wild type PDE11A and L756Q variant. Q756 mutant was predicted to induce more hydrogen bonds and then affect Helix structure. 
A

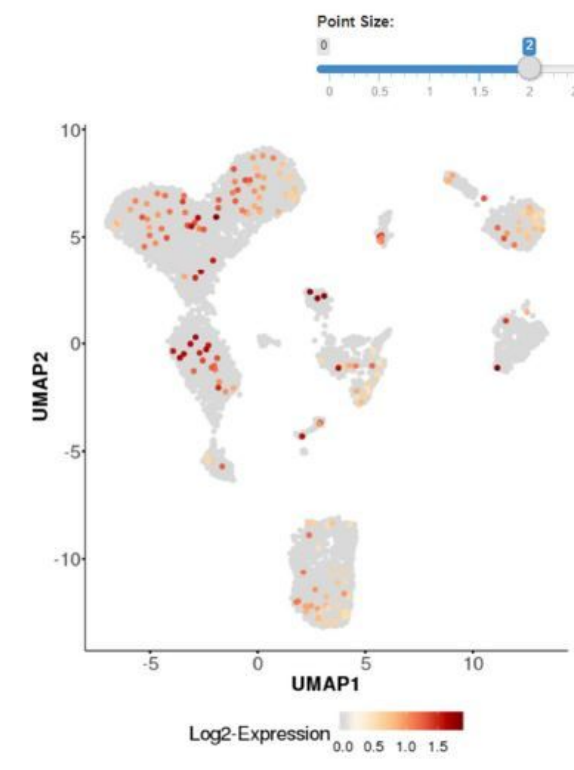

C

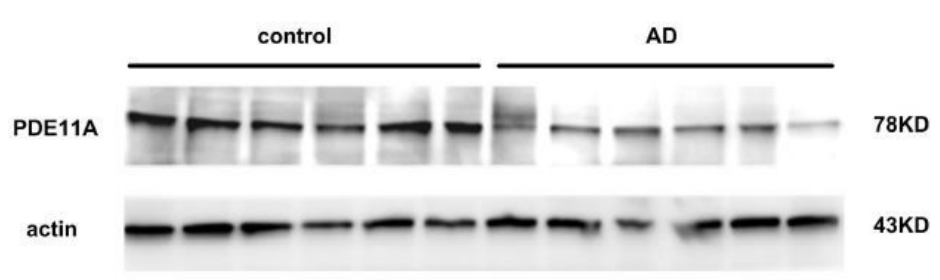

B
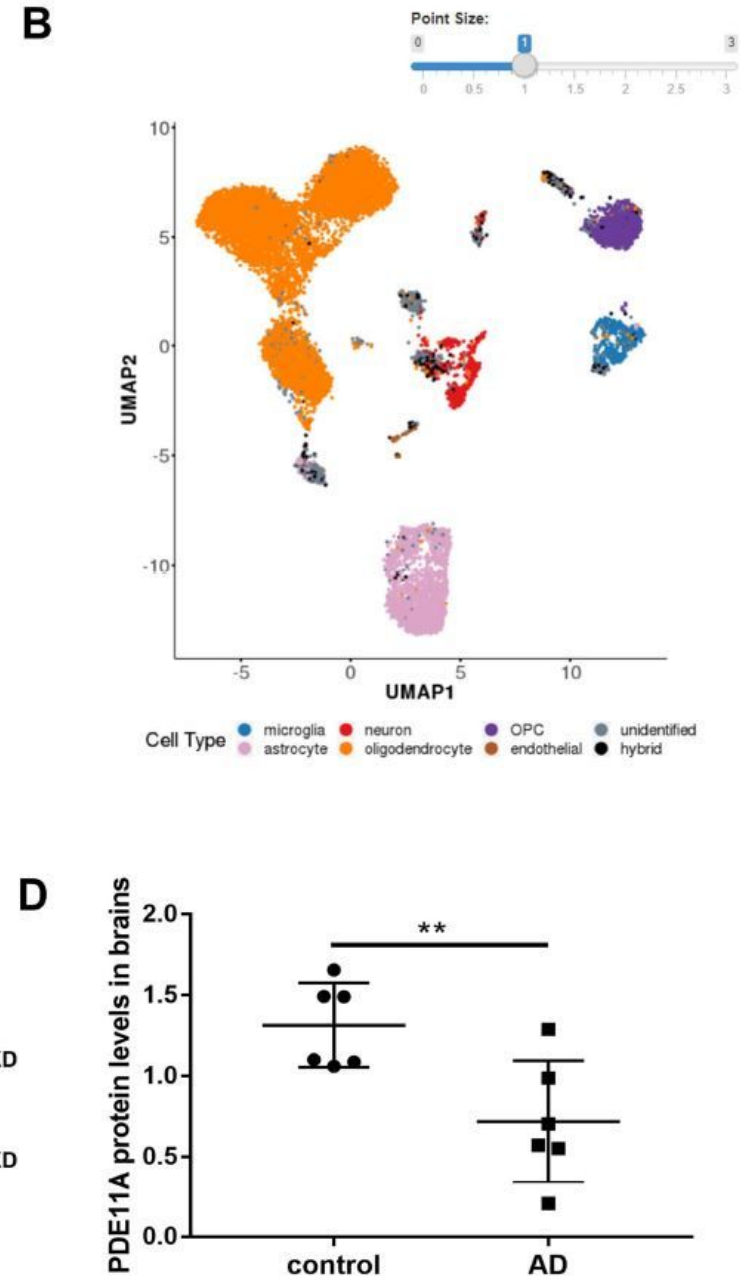

\section{Figure 3}

The PDE11A expression in brain tissues of AD. (A, B) Based on single-nucleus RNA sequencing data, PDE11A gene was expressed almost in all kinds of cells. Western blot $(C)$ and quantitative analysis (D) of PDE11A protein levels in post-mortem brain tissues. The data are represented as the mean \pm SEM, based on three unrelated measurements. ${ }^{*} \mathrm{p}<0.01$ by Student's t-test. 
A

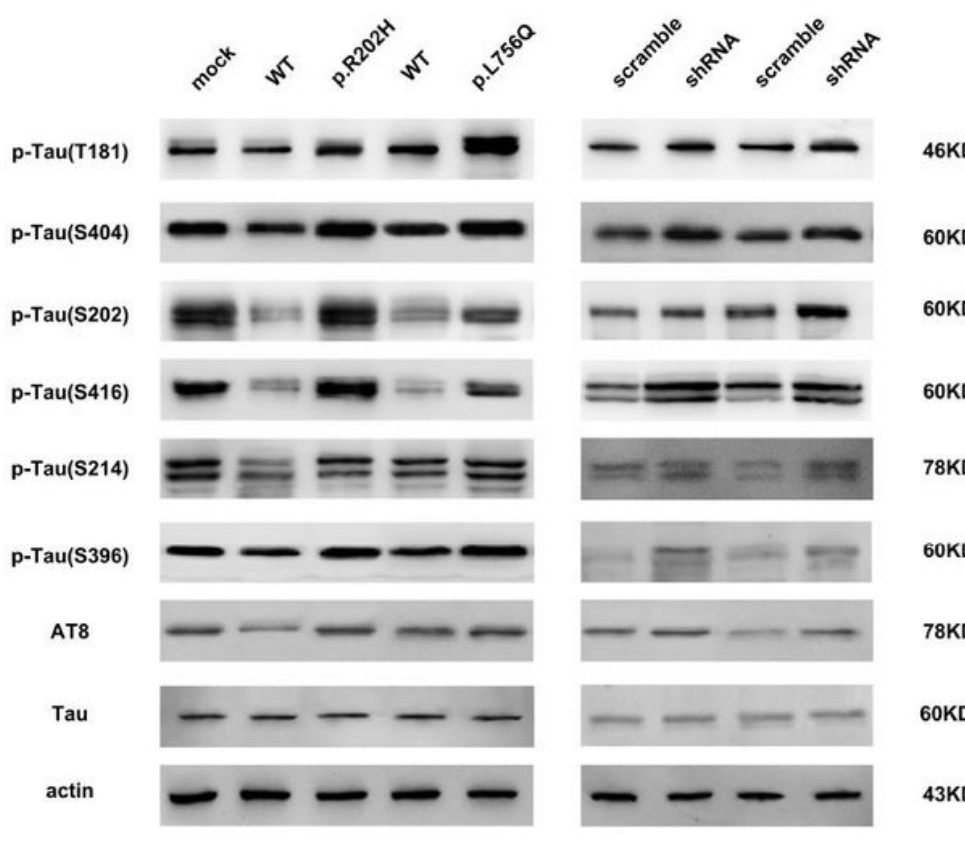

B

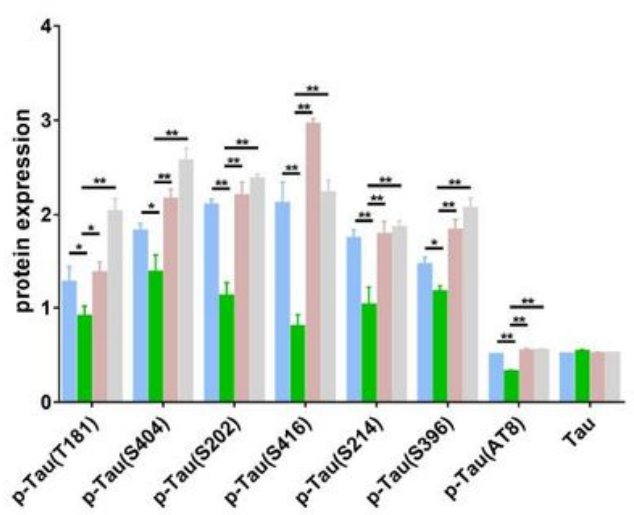

78KD

ОКD

C

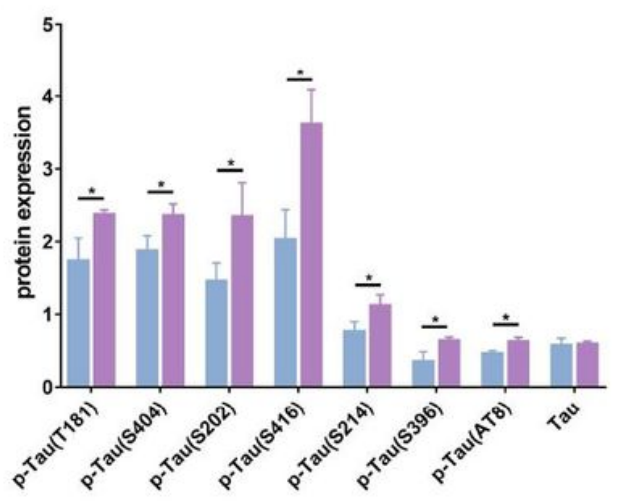

8KD

OKD mock WT

p.R202H

p.L756Q

scramble - ShRNA

\section{Figure 4}

The PDE11A variants led to significantly high Tau phosphorylation levels. The primary neurons were co-infected with MAPT and PDE11A lentivirus (PDE11A WT, mutants, scramble or shRNA). Seventy-two hours after infection, cell lysates were used to detect levels of p-Tau(T181), p-Tau(S404), p-Tau(S202), pTau(S416), p-Tau(S214), p-Tau(S396), p-Tau(AT8) and Tau. Western blot (A) and quantitative analysis (B, C) of phosphorylated Tau levels. The data are represented as the mean \pm SEM, based on three unrelated measurements. ${ }^{\star} p<0.05,{ }^{* \star} p<0.01$ by one-way ANOVA or Student's t-test.

A

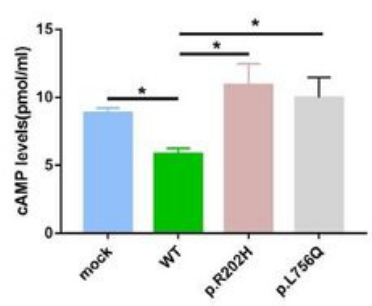

B

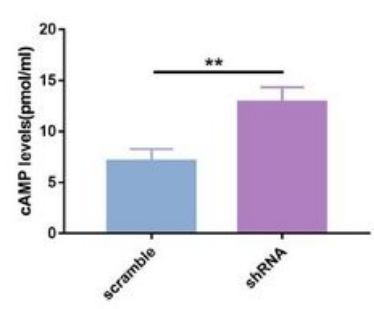

c
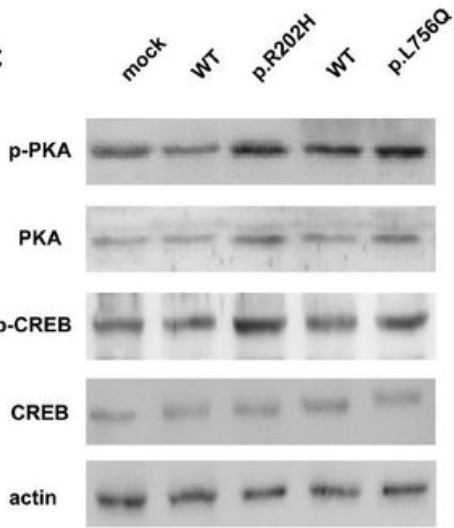
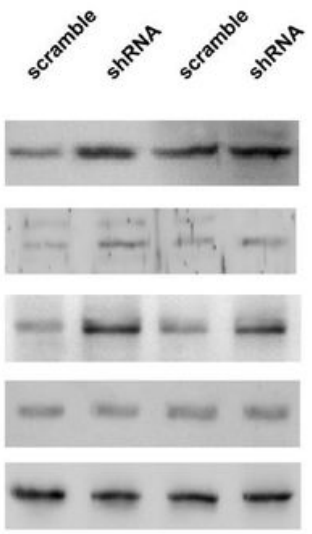

D

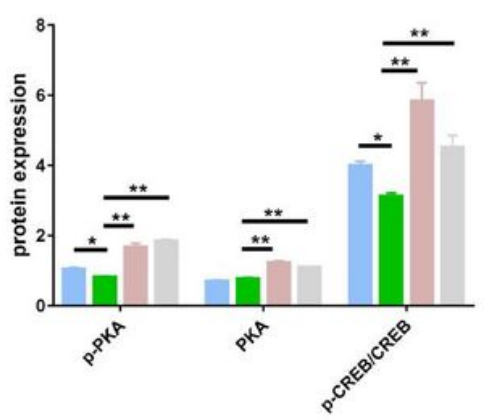

43KD

$40 K D$
$37 K D$
$40 K D$
$40 K D$
$43 K D$

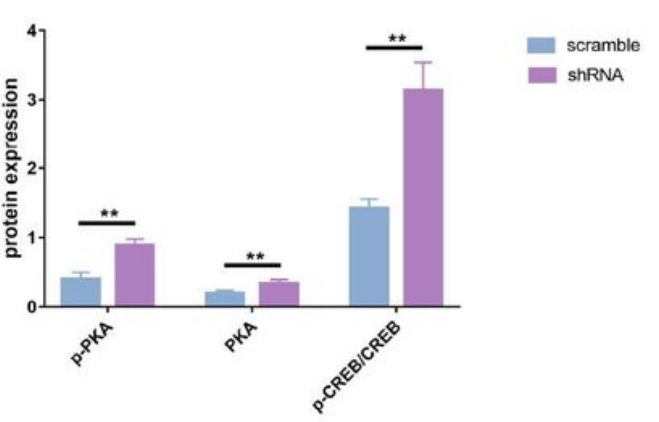


The PDE11A variants affect cAMP/PKA signaling. ELISA analysis showed cAMP levels in primary neurons after infection with WT PDE11A (with MAPT) decreased as expected; they increased following infection with PDE11A p.Arg202His, PDE11A p.Leu756Gln (A) or shRNA (with MAPT) (B). Western blot (C) of PKA signaling, and quantitative analysis of p-PKA, PKA, p-CREB/CREB in primary neurons infected with PDE11A mutants (D) or shRNA (with MAPT) (E). The data are represented as the mean \pm SEM. ${ }^{*} p<0.05$, ${ }^{\star *} p<0.01$ by one-way ANOVA or Student's t-test.

A

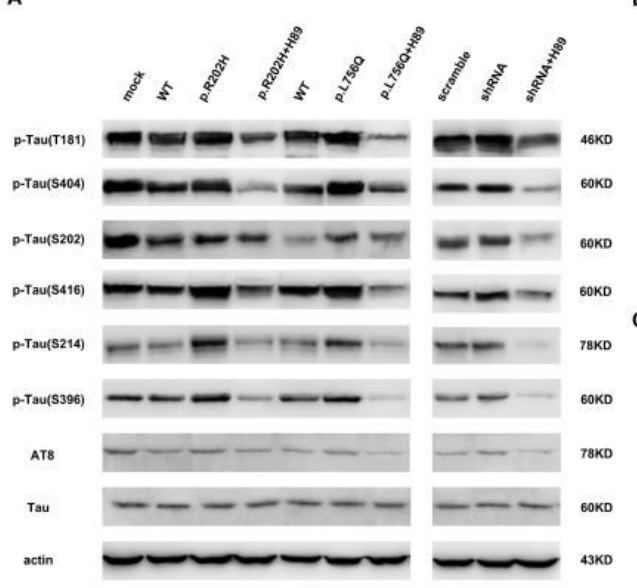

B
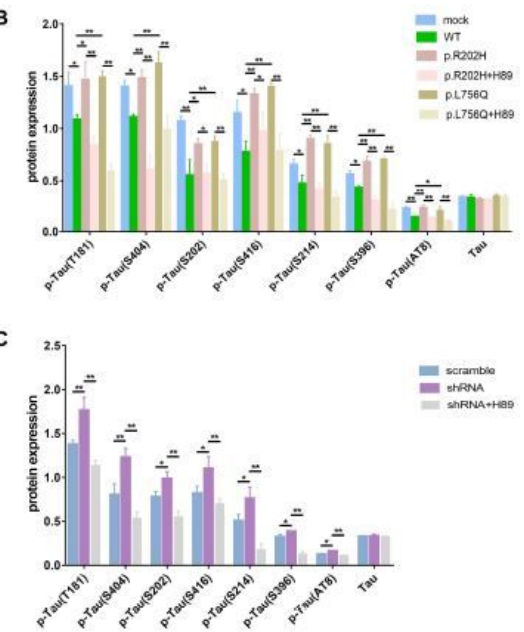

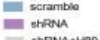

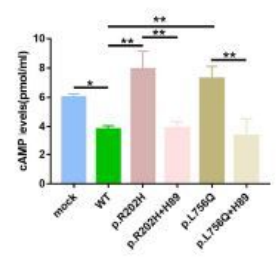

E

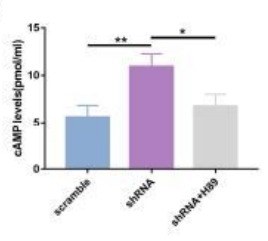

G
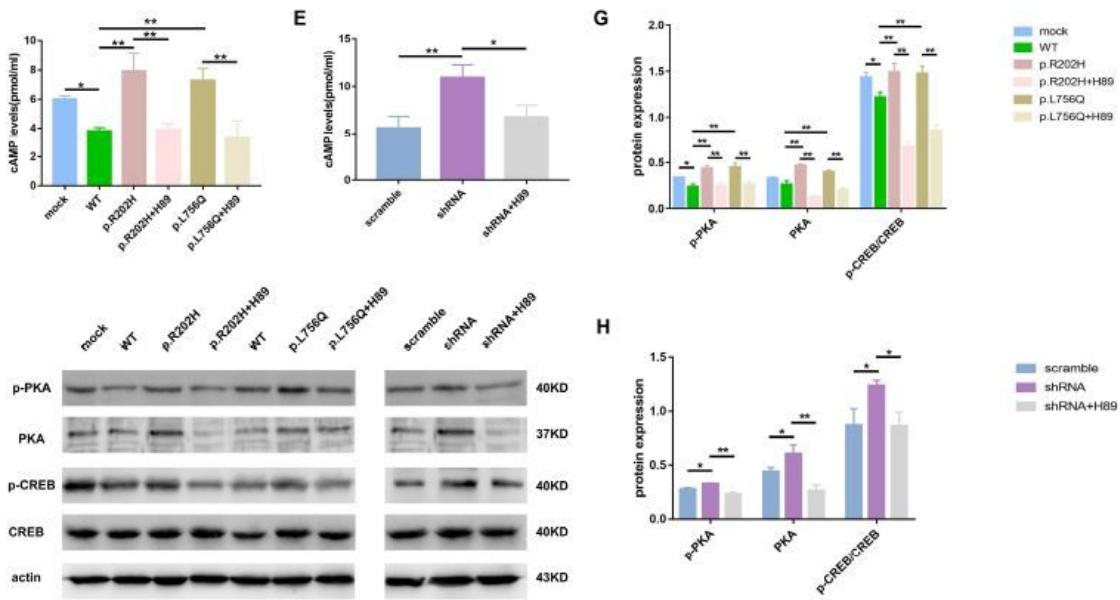

H

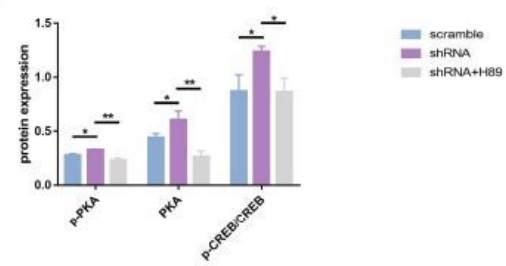

Figure 6

The effects of PDE11A mutants on phosphorylation of Tau and cAMP/PKA signaling after treatment with PKA inhibitor. HEK293 cells were treated with or without the PKA inhibitor H89 (10M). Then cells were co-transfected with MAPT and PDE11A mutant or shRNA plasmids. (A) Cell lysates were collected to examine Tau phosphorylation levels using Western blot. (B, C) Quantitative analysis of Tau phosphorylation levels. (D) ELISA analysis of cAMP levels. (E) Western blot of PKA/CREB signaling levels. $(F, G)$ Quantitative analysis of PKA/CREB signaling levels. The data are represented as the mean \pm SEM. ${ }^{*} p<0.05$, $* * p<0.01$ by one-way ANOVA.

\section{Supplementary Files}

This is a list of supplementary files associated with this preprint. Click to download.

- Additionalfile2.doc

- Additionalfile1new.xlsx 